\title{
A Simple Regression Based Heuristic for Learning Model Trees
}

\author{
Celine Vens and Hendrik Blockeel \\ K.U.Leuven - Department of Computer Science
}

\begin{abstract}
The term "model trees" is commonly used for regression trees that contain some non-trivial model in their leaves. Popular implementations of model tree learners build trees with linear regression models in their leaves. They use reduction of variance as a heuristic for selecting tests during the tree construction process. In this article, we show that systems employing this heuristic may exhibit pathological behaviour in some quite simple cases. This is not visible in the predictive accuracy of the tree, but it reduces its explanatory power. We propose an alternative heuristic that yields equally accurate but simpler trees with better explanatory power, and this at little or no additional computational cost. The resulting model tree induction algorithm is experimentally evaluated and compared with simpler and more complex approaches on a variety of synthetic and real world data sets.
\end{abstract}

Keywords: model trees, regression, heuristics, machine learning

\section{Introduction}

Induction of decision trees $[13,2]$ is a frequently used machine learning technique, not just for classification but also for numerical prediction (regression). Among regression trees, we can distinguish those that with each leaf associate a constant, as induced by for instance CART [2], and those that associate a less trivial model with each leaf $[1,7,8,10,14,15]$. For the latter we will use the term "model trees". A popular algorithm for building model trees with linear models in the leaves is M5 [14], of which M5' [17] is a re-implementation that is included in the well-known Weka software [19]. Another well-known approach is RETIS [8], which uses a more sophisticated heuristic.

The main difference between the M5' and RETIS approaches is that M5' first learns a standard regression tree (with constants in the leaves) and only afterwards, during a pruning phase, turns it into a model tree. RETIS aims immediately at building a model tree and uses a heuristic tuned towards this 


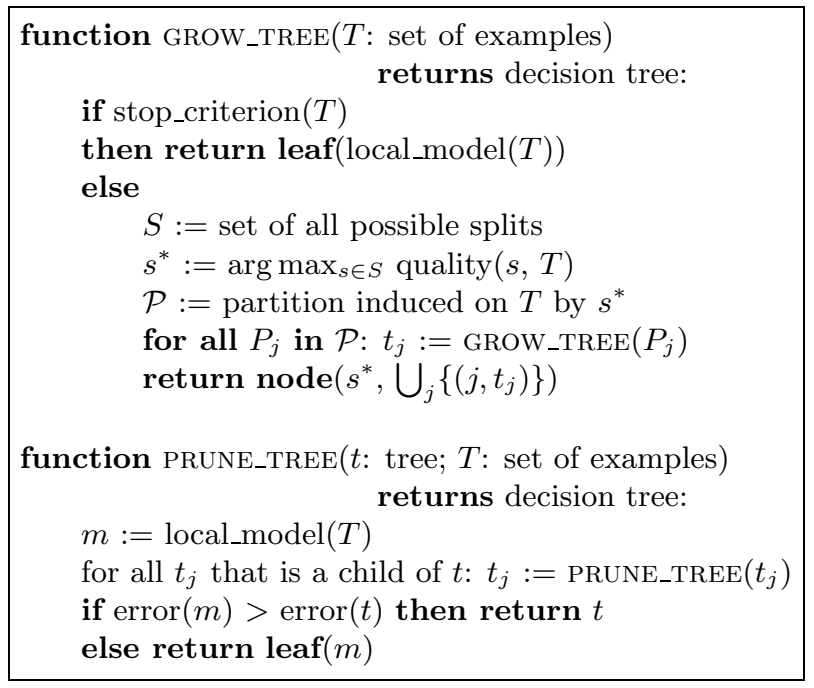

Figure 1: A generic TDIDT algorithm.

task. This heuristic, however, is quite expensive to compute, which may render the RETIS approach infeasible for certain practical problems.

M5', on the other hand, has other deficiencies. In Section 2, we discuss the variance based approach to model tree building and demonstrate its potential to exhibit pathological behaviour that strongly reduces the explanatory power of the induced trees. Section 3 discusses a number of systems that use a more complex heuristic. However, these systems have high computational complexity. This motivates us to look for an approach that is approximately as efficient as that of variance based systems, but avoids the pathological behaviour. We propose such a heuristic in Section 4. Section 5 presents experimental results, comparing our approach to existing ones. We conclude in Section 6.

\section{The Variance Based Approach}

Existing regression tree algorithms are instantiations of the "top-down induction of decision trees" algorithm (TDIDT); see Figure 1 for a generic variant. We refer to Breiman [2] or Quinlan [13] for full details on the TDIDT method. Roughly, the method recursively partitions the instance space such that "maximally homogeneous" (with respect to some target attribute) subregions are obtained, and recursively re-applies this procedure on the subregions until all subregions are sufficiently homogeneous or until further partitioning does not improve homogeneity any more. Subsets in the partition are always associated with specific attribute values, so that new instances can be assigned to the correct subregion by looking at their attribute values.

TDIDT algorithms vary mainly with respect to the heuristic they use to 


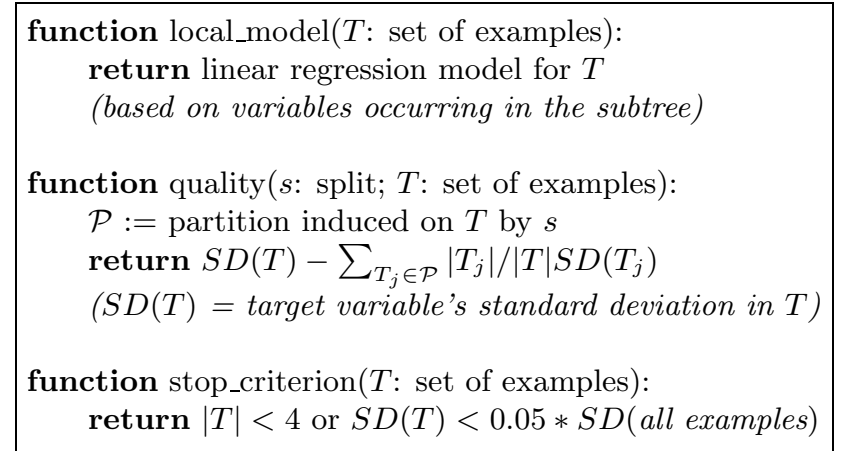

Figure 2: M5' instantiation of TDIDT.

decide which partitioning is best and the model that is stored in each leaf. For instance, CART [2], when building regression trees, uses variance reduction as a heuristic (that is, it tries to reduce the variance of the target variable within the subsets as much as possible), and the model stored in a leaf is the mean of the target values of all examples in that leaf. M5' [17] also uses variance ${ }^{1}$ reduction as a heuristic, and fills in constants in the leaves, but in its pruning phase it changes internal nodes into leaves containing a linear model if that model performs at least equally good as the subtree rooted in that node. The linear model uses as predictor variables only attributes occurring in the subtree that it replaces. Hence, M5' can be described as an instantiation of the generic TDIDT algorithm. Figure 2 shows the instantiation of the local_model, quality, and stop_criterion functions.

Keeping in mind that linear models will be built in the leaves, it seems reasonable to select tests that maximize the expected quality of these models. In that sense, variance reduction is not a very suitable heuristic. As also noted elsewhere $[8,10]$, the quality of a linear model constructed for a data set is quite independent of the variance of that data set. For instance, a set of points that form a perfectly linear but steep line has a greater variance than a set of points closer together but randomly distributed. Given this independence, there is no reason why variance would perform better than random splitting. In fact, it may even be worse, as the following simple example shows.

Consider the piecewise linear function $y=x, 0 \leq x \leq 1 ; y=2-x, 1<x \leq 2$ (see Fig. 3(a)). Clearly the simplest model tree to represent this function is one that splits on the condition $x<1$ and builds two linear models.

In the following, we consider a continuous uniform distribution of $(x, y)$ couples. Note that for the optimal tree, the variance along $y$ of both subsets is equal to the variance along $y$ of the original set; thus, from the point of view of reducing variance, this tree would not be considered a good tree. The split

\footnotetext{
${ }^{1}$ Or variations of variance, such as standard deviation [17] or the 5 th root of the variance, as in the Weka implementation.
} 


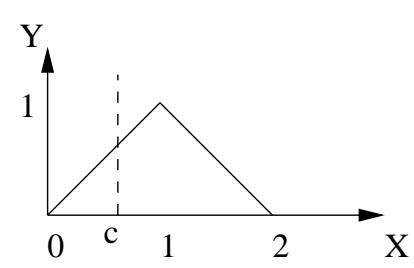

(a)

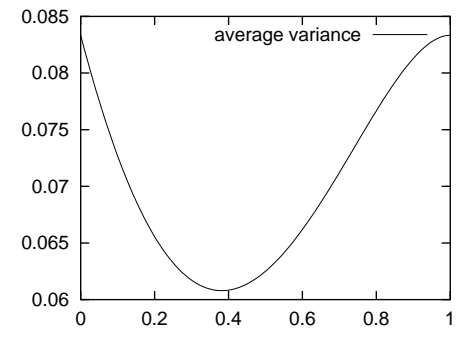

(b)

Figure 3: (a) A piecewise linear function and split point $c$. (b) The weighted mean of the variance in the subsets defined by $x \leq c$ and $x>c$ as a function of $c$.

$x<c$ that is found by M5' is the one that minimizes the sum of the weighted variance over both subsets. For a continuous uniform distribution, the optimal $c$ can be computed by minimizing $h(c)=c \cdot \operatorname{Var}(y \mid x \leq c)+(2-c) \cdot \operatorname{Var}(y \mid x>c)$, where the factors $c$ and $2-c$ are weights referring to the respective sizes of the subsets and the Var factors are the variances of these subsets. The optimal $c$ is then defined as $c^{*}=\arg \min _{c} h(c)$. Due to symmetry, $2-c^{*}$ is an optimum in the interval $[1,2]$.

The function $h(c)$ is plotted in Fig. 3(b), and there it can clearly be seen that a minimum is obtained near 0.4 ; that is, quite far away from the optimal split point 1 . In fact, even random splitting can be expected to yield slightly better results on average, since it has $60 \%$ probability of creating a split closer to 1 .

Without loss of generality, given the symmetry, we assume $c^{*}<1$. The left subset yields a perfect linear model. However, as the model is not actually constructed as part of the stopping criterion, M5' constructs a subtree for this subset, partitioning this linear area into many small areas with ever decreasing variance; only in the pruning phase does it remove this subtree to build the linear model. The right subset is a function similar to the original one, though less symmetric. Hence, similar behaviour can be expected. Note that in contrast to the left branch, the right branch cannot be pruned to a single leaf; and that also further down the tree, the splits will always be off the optimal point. Thus a relatively deep tree will still remain.

The above reasoning shows that variance-based heuristics tend to split the data set in the wrong places. This reduces the explanatory power of the tree: first, a larger tree is obtained than is necessary, and second, the splits in the tree are not really informative. Moreover, superfluous partitioning of the data space causes relatively small areas to be separated, with each area containing 
few examples. As local models built from few examples tend to be less accurate, the predictive performance of the induced trees may also be reduced.

In our experiments section we further explore this behaviour of M5'. At this point, we just illustrate the concrete behaviour of M5' on the simple function mentioned above, for a random sample of data points with some noise added. Figure 4 shows the tree M5' builds for such a data set. It confirms our theoretical analysis: the model built by $\mathrm{M}^{\prime}$ ' is good in the sense that it has a reasonably good predictive performance, but its explanatory power is diminished.

Another system that uses variance reduction as a heuristic is HTL [15]. The major difference with M5 is that it is a hybrid system that can integrate several alternative models into the tree leaves. However, since the heuristic that we will present in Sect. 4 provides a solution only in the case where linear models are used in the leaves, we will not elaborate on HTL.

\section{More Complex Approaches}

Our simple case not only shows that variance does not work very well as a heuristic, it also suggests that constructing linear models for the subsets and evaluating their goodness of fit should work fine. This is exactly the approach followed by Retis [8]. ReTis evaluates a split by building a multiple linear model for each subset and computing its residual variance. Also in the leaves multiple linear regression models are built. The RETIS instantiation of TDIDT is shown in Figure 5. Unfortunately, these multiple regressions result in a complexity that will not scale up to large problems. Torgo [16] has presented a set of incremental formulae that allows to evaluate the set of candidate splits for a given variable with complexity quadratic in the number of attributes. Hence, the complexity for evaluating all splits in a node is cubic in the number of attributes, which is still too high for many practical applications. Besides its high computational cost, another drawback of RETIS is that only full regression models (i.e. regression models in all variables) are considered in the leaves and in the quality function. Next to the obvious fact that understanding a smaller model is usually easier than understanding a larger one, it is well-known that if some of the predictors are related to each other, that is, they are (approximately) collinear, models based on subsets may give more precise results than will models based on more variables [18]. Therefore, variable selection is a desirable part of many regression analyses. In the case of RETIS, applying variable selection techniques when choosing the best split may become too costly, since it may require the computation of a high number of multiple regression models [10].

A number of systems have been proposed that were designed to address the computational concerns of RETIS. They can be divided into two groups. The first group uses multiple linear regression models in the leaves, but at each node transforms the regression problem into a classification problem in order to use more efficient search strategies. Examples are Support [4], GUIDE [9], and SECRET [6]. The first two systems make use of statistical methods for variable 


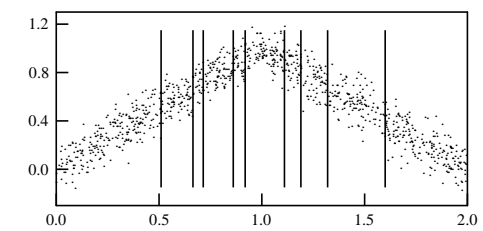

(a)

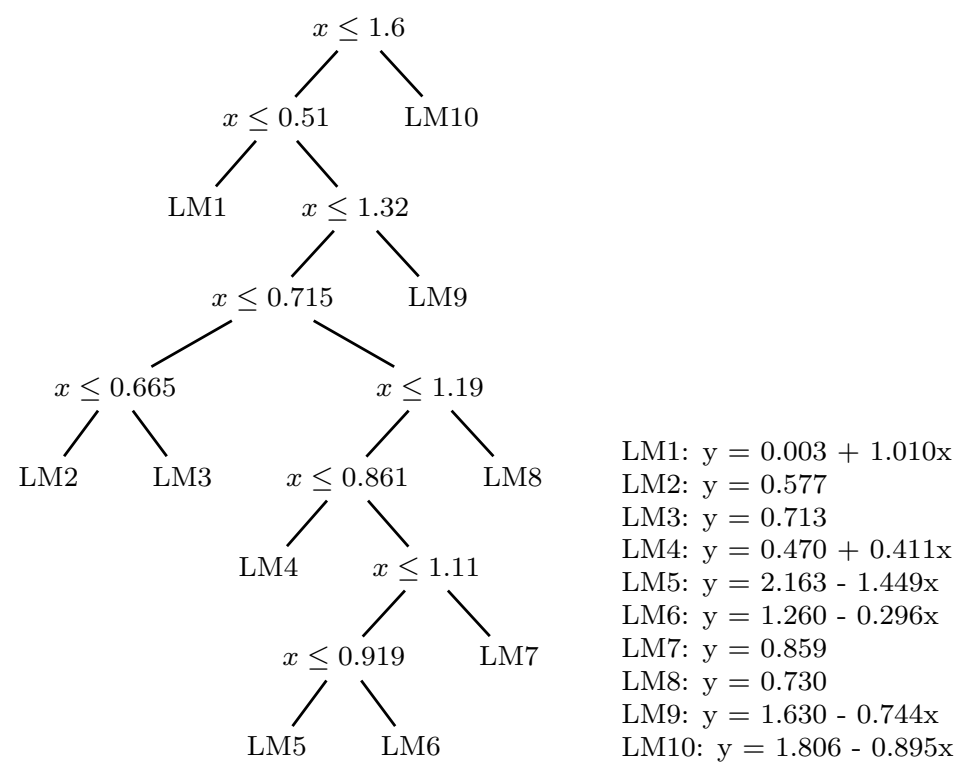

(b)

Figure 4: (a) A simple data set. The vertical lines in the data plot indicate where the split points of a variance based model are. (b) The model induced for this dataset by a variance based model tree builder. 


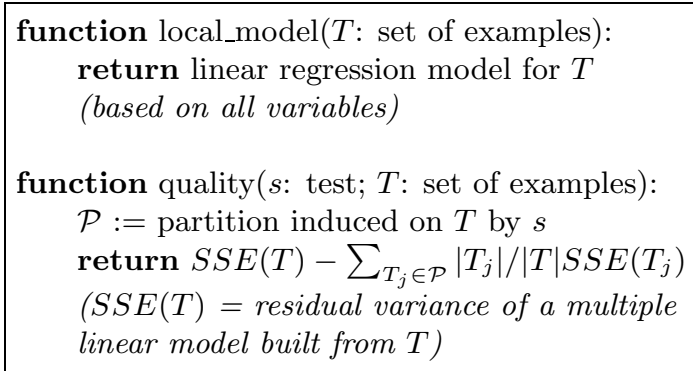

Figure 5: RETIS instantiation of TDIDT.

selection. At each node, a (constant or linear) model is fit, then the signs of their residuals are used to separate the observations into two classes and a statistical test is used to pick the variable giving the largest separation between the two classes. The two systems differ in the test they use and in the way to determine the split point, once the variable is found. However, as Dobra and Gehrke [6] point out, these approaches may also lead to redundant splits. SECRET uses the EM algorithm at each node to determine two Gaussian clusters in the data and to label the data based on the closeness to these clusters. Classification tree techniques are then used to select the split attribute and the split point.

The second group retains the regression problem at each node, but fits a simpler regression model to evaluate a split. In this paper, we will focus on the second group. Obviously, M5 is an example of this approach, since it fits the most basic model at the nodes: the average of the sample data at that node.

The simple example described in the previous section deals with a single predictor variable, which is uncommon. Consequently, the variable used to define the split is the same as the variable used in the regression. When multiple predictor variables exist, a number of different approaches are possible, with RETIS and M5 at both ends of the spectrum. We characterize each of them by listing the variables for which it performs regression in order to evaluate the split (not to be confused with the construction of linear models in the leaves), given the variable it splits on. We consider only univariate splits here. All these options could be extended by considering also multivariate splits [3], but this extension is more or less orthogonal to the dimension discussed here, and hence out of scope for us.

The four options we distinguish are:

1. no regression (the M5 approach)

2. simple regression on the split attribute if it is numerical

3. simple regression on all numerical attributes separately

4. multiple regression on all numerical attributes together (the RETIS approach) 


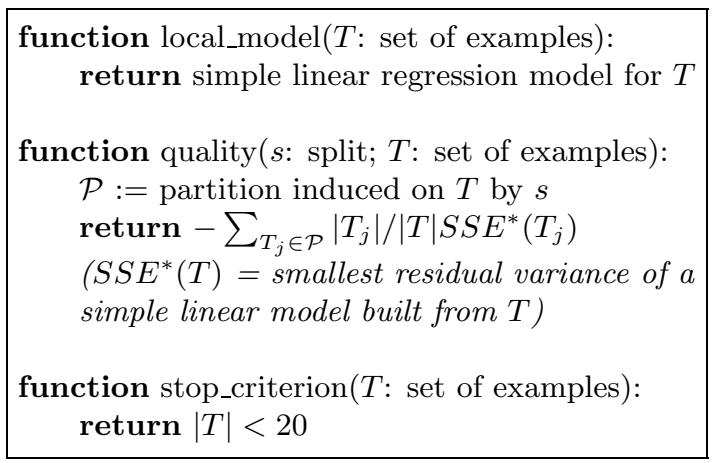

Figure 6: TREed REgRESSION instantiation of TDIDT.

Our reason for listing exactly these different options is that they differ significantly with respect to their computational complexity: options 1 and 2 are linear in the number of attributes, option 3 quadratic and option 4 cubic. All of these complexity factors are multiplied with at least $N$, with $N$ the number of examples.

An example of a system that takes the third option is described by Alexander and Grimshaw [1] and is called TREed REgREssion. To evaluate a split, say, on attribute $X_{j}$, each independent attribute is evaluated as the regressor attribute for a simple regression. The best linear model is determined for each child node independently. The algorithm stores simple linear regression models at each leaf. The TDIDT instantiation is shown in Fig. 6.

Another example of an option 3 system is SMOTI [10]. It induces an alternative kind of decision trees, where besides splitting nodes, regression nodes can be introduced. Whereas the former partition the sample space, the latter perform only simple linear regression. The regression is done in such a way that the multiple linear model associated to each leaf is obtained by composing the effect of regression nodes along the path from the root to the leaf. The purpose of this tree building mechanism is to discover global effects that some attributes may have in the underlying model function. In order to evaluate a split, simple regression is performed on all attributes separately and combined with the regressions associated to regression nodes along the path from the root to the node. In order to evaluate a regression node, a lookahead step is performed, which actually renders this method cubic in the number of attributes [10].

\section{A Simple Linear Regression Based Heuristic}

In this section we present an algorithm that uses a simple linear regression based heuristic, hence it follows option 2 from the previous section. We start by motivating our approach in section 4.1 and discuss the algorithm in section 4.2 . 


\subsection{Motivation}

To our knowledge, there exists no system that uses option 2, although such a system would have some interesting properties:

- It is expected to provide a solution for the undesirable behaviour that systems using option 1 exhibit. More specifically, it should work better in those cases where at least one variable has an influence on the target variable that is non-linear but can be approximated with a piecewise linear function.

- It is consistent with an idea that underlies most univariate decision tree methods: evaluating the predictive power of each attribute independently. Option 3 boils down to splitting on one attribute and examining how this influences the predictive power of other attributes; similarly to the use of lookahead in decision tree building, an approach advised against [12].

- It has a computational complexity close to that of option 1 , differing only with a constant factor. Variance, $\sigma^{2}=\sum_{i}\left(y_{i}-\bar{y}\right)^{2} / n$ with $\bar{y}$ the mean of the $y_{i}$, can be computed entirely from the sufficient statistics $\sum_{i}\left(1, y_{i}, y_{i}^{2}\right)$ with $i$ varying over all elements of the data set. Similarly, a simple linear regression model as well as its residual variance can be computed from $\sum_{i}\left(1, x_{i}, x_{i}^{2}, y_{i}, y_{i}^{2}, x_{i} y_{i}\right)$. This takes about three times longer. Computing the residual variance from these statistics is about four times as much work as computing the total variance from them. Hence, this heuristic is roughly three to four times as expensive to compute as the one in M5. In fact, since smaller trees can be expected, the slightly more complex computation might well be compensated.

Obviously, for each of the options 1-3 it is possible to point out situations where the next more complex option performs better. The point that we wish to make, is that the move from option 1 to 2 solves at least some problems, and costs almost nothing with respect to efficiency; hence there appears to be no good reason for preferring option 1 over option 2 .

\subsection{The algorithm}

The model tree induction algorithm we propose follows option 2 and is a variant of M5' that we call MAUvE ("M5' Adapted to use Uni-VariatE regression"). The MAUve instantiation of TDIDT is shown in Figure 7. The main difference with M5' lies in the heuristic, which is based on simple regression. Also the stop criterion and pruning method are altered to be better tuned towards the heuristic. We discuss each of these, as well as other components of the algorithm (pruning and smoothing).

\subsubsection{Quality function}

A model tree is built by calling the GROW_TREE function (see Figure 1) using all examples. For each input variable $X_{j}$, we pass through the possible split 


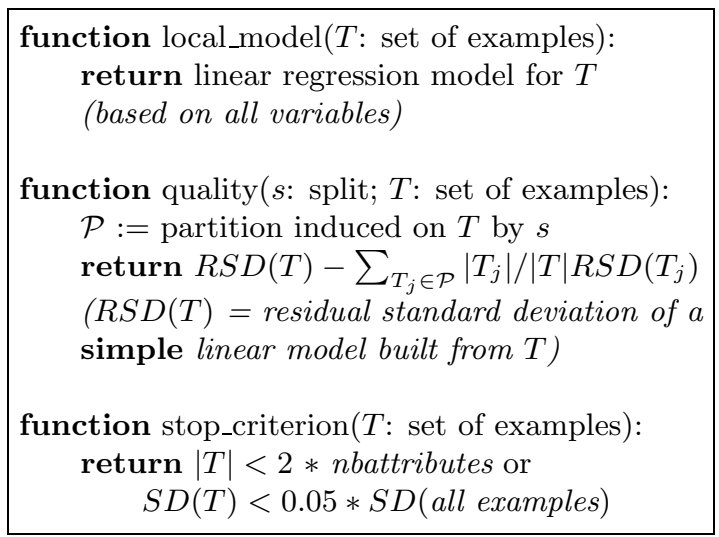

Figure 7: MAUVE instantiation of TDIDT.

points. If $X_{j}$ is numeric, each possible split takes the form $X_{j}<v$ with $v$ an element from the domain of $X_{j}$. The splits are evaluated by calculating, for each subset of the partition induced by the split, the residual standard deviation of the target variable after fitting a simple linear regression model with $X_{j}$ as regressor.

If $X_{j}$ is a nominal variable, say it takes values in $V=\left\{X_{j 1}, X_{j 2}, \ldots, X_{j m},\right\}$, then splits take the form $X_{j} \in V^{\prime}$ with $V^{\prime} \subset V$, i.e. we consider only binary splits. Each of these splits can be seen as testing the value of a binary variable (a variable that has value 1 for those examples with $X_{j} \in V^{\prime}$, and value 0 otherwise). Since the residual standard deviation is undefined when using binary variables, these splits are evaluated using the (normal) standard deviation of the target variable. If $X_{j}$ can take $m$ possible values, there are $2^{m-1}$ different binary partitions possible, so we need to consider $2^{m-1}$ binary variables. However, since the binary splits minimize the (normal) standard deviation, we can make use of a result by Breiman [2] to reduce the number of binary splits to be considered to $m-1$, as in the implementation of M5'. To this purpose, the average target value corresponding to each possible value for $X_{j}$ is computed and the values in the enumeration are sorted according to these averages. Then $X_{j}$ is replaced by $m-1$ binary attributes, the $i$ th being 0 if the value is one of the first $i$ in the ordering, and 1 otherwise.

For each input variable $X_{j}$, the calculations of the (residual) standard deviation are performed incrementally, by sorting the examples by their $X_{j}$-value, and by updating a set of statistics $\sum_{i}\left(1, x_{i}, x_{i}^{2}, y_{i}, y_{i}^{2}, x_{i} y_{i}\right)$ for each possible split point. From all possible splits, we choose the one that maximizes the reduction in (residual) standard deviation. The function is recursively applied to the subsets of the partition induced by the chosen split. 


\subsubsection{Local models}

The occurrence of a certain split attribute $X_{j}$ at a node $N$ reflects the existence of a linear relation between the target and $X_{j}$ in the child nodes of $N$. Hence, $X_{j}$ should be included in the linear model of the leaves in the subtree of $N$. Since attributes not occurring on a particular path may also be relevant, the model stored in each leaf is a multiple linear regression model that considers all input attributes as predictor variables. As in M5', variable selection techniques are applied, in order to increase the reliability of the estimated regression coefficients, and hence to minimize the difference between predicted target and actual target.

\subsubsection{Stop criterion}

The stop criterion of the GROW_TREE function was slightly modified. M5' doesn't split a node if it contains less than four instances, because the minimum number of instances in a leaf is two. However, when building a linear model in $k$ variables in the leaves, one needs at least $k+1$ examples. Taking into account that we consider all predictor variables to be included in the model, we need at least a number of examples equal to the number of predictors plus one, i.e. the total number of attributes, in each leaf. Hence, at least 2 times the number of attributes are needed to split a node. The second part of M5's stop criterion (see Fig. 2) was left unchanged.

\subsubsection{Pruning}

In the pruning phase, each interior node $N$ is associated with two error measures. The first is called the model error $M E(N)$ and is the error that would be experienced if the node were a leaf (hence, a multiple regression model is built at the node). The second is the subtree error $S E(N)$ and measures the error if the node keeps its child nodes. The tree is pruned at $N$ (i.e. its subtrees are discarded and the node becomes a leaf) if $M E(N)<S E(N)$. In order to compensate for the underestimation of these errors on unseen cases, they are multiplied by $(n+m * p) /(n-p)$ with $n$ the number of examples reaching the node $N, p$ the number of parameters, and $m$ the pruning factor, a multiplier that controls the size of the tree [17]. The number of parameters is the sum of the number of nodes in the subtree starting at $N$ and the number of variables occurring in the linear equations of the leaves in the same subtree. The pruning factor $m$ has default value 2 in M5', which is taken over in MAUVE. Of course, for both systems, performance can be improved by using crossvalidation to estimate an optimal value (see Sect. 5.2.2).

\subsubsection{Smoothing}

Smoothing [14] is a method for improving the predictions of a model tree. It can be used in the final stage of the tree building process. It compensates for the sharp discontinuities that may occur between adjacent linear models at the 
leaves of the pruned tree. A weighted average between models in the leaves and linear models higher up in the tree is taken to make predictions. Smoothing may substantially increase the accuracy of predictions, without altering tree size.

\section{Experimental Results}

This section serves to evaluate the performance of our system and to compare it with traditional variance based approaches and with more sophisticated approaches. In particular, three questions are addressed:

1. If there is a (piecewise) linear relation between the target and (at least) one of the input variables, does our approach perform better than a variance based approach?

2. How often does such a linear relation occur in practice?

3. Does the use of more sophisticated approaches result in a performance improvement?

In order to answer the first question, experiments were performed on synthetic data sets, specifically constructed with (piecewise) linear relations (Section 5.1). The second question is tackled by conducting experiments on real world data sets (Section 5.2). The third question is dealt with by performing a more complete comparison of our approach to other existing approaches on the same real world data sets (Section 5.3).

All experiments were performed using tenfold crossvalidation and were run twenty times in order to account for the variance between the folds. The relevant criteria are predictive performance, tree size, and induction time. Predictive performance is estimated using the RRMSE ${ }^{2}$. Tree size is measured in number of leaves. Since the number of leaves alone does not give a good estimation of the complexity of the model (which is also influenced by the number of variables in the linear equation in the leaves), we also report the number of parameters. The number of parameters is the sum of the number of nodes in the tree and the number of variables occurring in the equation in each leaf.

For our experiments, standard settings in Weka were used, except for smoothing, which was initially switched off.

\subsection{Synthetic Data Sets}

Figure 8 shows some simple functions, in which there is a piecewise linear relation (or a relation that can be approximated by it) between the target and the input variable. For each function $f(x)$, a data set was constructed by drawing a random sample of 1000 values from a uniform distribution over $x$, and associating with each $x$ a $y=f(x)+\epsilon$ with $\epsilon$ a normally distributed random variable

\footnotetext{
${ }^{2} \mathrm{RRMSE}=$ root relative mean squared error, this is the root of the ratio of the mean squared error of the tree to the mean squared error of a trivial model always predicting the mean.
} 

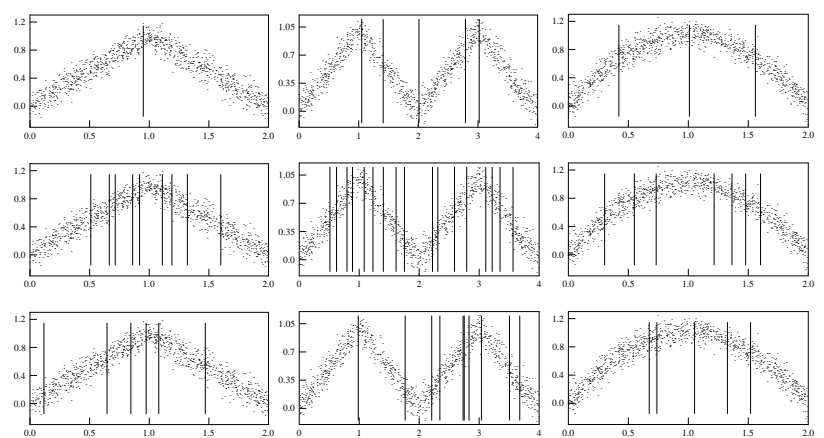

Figure 8: Approximating functions of one variable. Three different functions are shown, we call them (from left to right) function1, function2 and function3; for each we indicate the thresholds that are created by (from top to bottom) MAUVE; M5' with its original variance heuristic; M5' with random splits.

Table 1: Predictive accuracy (RRMSE) for synthetic datasets when using simple regression (MAUVE), variance (M5'), and random heuristics (RND).

\begin{tabular}{cccc}
\hline & MAUVE & M5 & RND \\
Data set & RRMSE (StdDev) & RRMSE (StdDev) & RRMSE (StdDev) \\
\hline function1 & $\mathbf{3 3 . 9 9 \% ( 0 . 0 4 )}$ & $34.35 \%(0.12)$ & $34.39 \%(0.22)$ \\
function2 & $\mathbf{3 2 . 9 6 \% ( 0 . 1 3 )}$ & $34.72 \%(0.21)$ & $33.61 \%(0.37)$ \\
function3 & $\mathbf{3 1 . 3 9 \% ( 0 . 0 6 )}$ & $31.70 \%(0.20)$ & $31.63 \%(0.53)$ \\
function4 & $\mathbf{1 6 . 4 0 \% ( 3 . 0 5 )}$ & $21.83 \%(1.85)$ & $68.64 \%(2.97)$ \\
\hline
\end{tabular}

with mean 0 and standard deviation $\sigma$ that represents noise. Varying $\sigma$ had no significant influence on the interpretation of our results.

For each function, Figure 8 shows the partitioning created by model tree builders using simple regression, variance, and random splits. These results confirm our earlier findings: for a variety of functions, MAUvE tends to find simpler models, with fewer split points that intuitively make more sense. Introducing random splits works approximately as well as using variance-based splits, or even slightly better.

We have also constructed piecewise linear functions in multiple predictor variables. Figure 9 presents a representative example problem. A dataset of 700 examples was constructed according to the target model in Fig. 9(a), this time no noise was added. From Fig. 9(b) and Fig. 9(c), we see again that MAuve produces a model that is much shorter than that of M5'.

Some statistics on the induced trees are presented in Tables 1 and 2. They confirm that the models induced by MAUvE are always simpler without causing any loss of accuracy. In fact, for these datasets, predictive performance is improved. Moreover, the induction times for MAUvE are similar to those of M5', which suggests that the tree building procedure stops earlier and that this effect compensates for the more complex heuristic. 


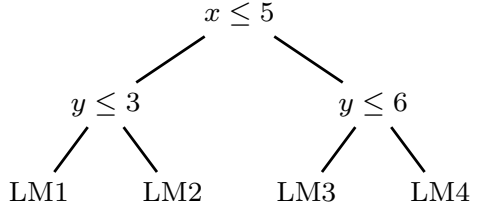

(a) Target model

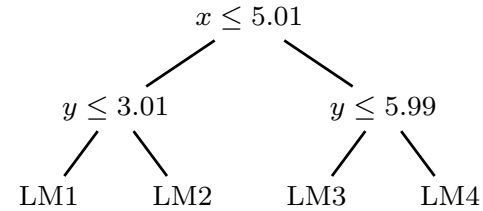

(b) MAuve's model

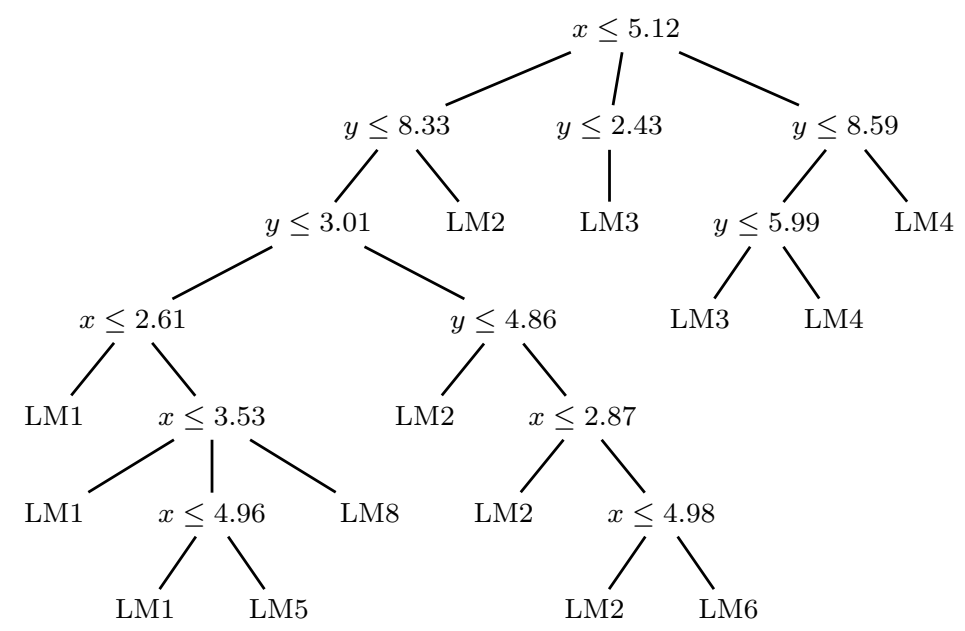

(c) M5's model

Figure 9: Learning a piecewise linear function of two variables (function4). (a) The original function. (b) The model tree built by Mauve. (c) The model tree built by M5'.

Table 2: Tree size and CPU times for synthetic datasets when using simple regression (MAUVE), variance (M5'), and random heuristics (RND).

\begin{tabular}{lrlrlrllll}
\hline & \multicolumn{4}{c}{ Size } & \multicolumn{4}{c}{ CPU time } \\
\cline { 2 - 10 } Data set & \multicolumn{1}{c}{ MAUVE } & \multicolumn{2}{c}{ M5' } & \multicolumn{2}{c}{ RND } & MAUVE & M5' & RND \\
& Leaves & Pars & Leaves & Pars & Leaves & Pars & Secs & Secs & Secs \\
\hline function1 & $\mathbf{2}$ & $\mathbf{5}$ & 10 & 25 & 5 & 13 & 3.75 & 3.41 & 3.87 \\
function2 & $\mathbf{6}$ & $\mathbf{1 7}$ & 18 & 44 & 12 & 32 & 3.95 & 3.51 & 3.45 \\
function3 & $\mathbf{4}$ & $\mathbf{1 1}$ & 8 & 20 & 6 & 16 & 3.11 & 3.95 & 2.89 \\
function4 & $\mathbf{4}$ & $\mathbf{1 5}$ & 13 & 47 & 9 & 28 & 1.67 & 1.51 & 2.23 \\
\hline
\end{tabular}


We conclude that, if we know that there is a simple piecewise linear relation (or a relation that can be approximated by it, such as function3) between the target attribute and (at least) one of the input attributes, MAUVE outperforms variance based methods. The question remains whether this linear behaviour also occurs in real life data sets.

\subsection{Real World Data Sets}

We have compared the performance of the different heuristics on a number of UCI data sets [11] and on some datasets taken from the collection of regression datasets by Luis Torgo ${ }^{3}$. Most of these datasets have been used as benchmarks in other studies on regression trees and model trees. For each dataset, we list the number of examples, the number of predictor attributes, the number of numeric predictor attributes, and the origin in Table 3.

\subsubsection{Standard experiments}

In this section, we compare the basic versions of M5' and MAUVE, i.e., with a fixed pruning factor (having M5's default value of 2) and without using the smoothing operator. Alternative parameter settings will be discussed in the following sections.

Table 4 shows predictive accuracies. Due to the high variation in the RRMSE, we also compared the error for MAUVE and M5' for each random seed of the crossvalidation and report the number of times each system won. As noted by Dietterich [5], the use of significance tests based on the means and standard deviations from repeated crossvalidations is not valid, since null hypotheses will nearly always be rejected given enough repetitions. Therefore, we don't make any statements regarding significance. Instead, we emphasize the results in Table 4 if one method outperforms the other one in at least $90 \%$ of the cases. Model complexity and timings are shown in Table 5. In that table, sizes are emphasized if one method results in a model that is at least one third shorter than the other method's model.

The results can be summarized as follows (see also Table 6). Overall, the predictive performance of MAUVE is better than, or equally good as M5'. In the one case where M5' performs clearly better (Auto-Price), the model output by MAUve is shorter. Concerning model complexity, in almost all cases, the tree found by MAUVE is smaller than, or equally large as the tree output by M5'. The only exception is Auto-Mpg, for which MAUve outputs a slightly larger tree than M5'. In Table 6 we see that, taking into account both accuracy and number of leaves, MAUVE has seven clear winners, against one for M5'.

If we look at the number of parameters, then the results are not that clear (not visible in Table 6). That MAUve is not longer outperforming M5' on this measure is probably due to the fact that it considers all predictor variables in the leaf models.

\footnotetext{
3 http://www.liacc.up.pt/ ltorgo/Regression/DataSets.html
} 
Table 3: Description of real world data sets.

\begin{tabular}{rccl}
\hline Data set & Examples & Attributes & Origin \\
\hline Housing & 506 & 13 (12 num.) & UCI \\
Machines & 209 & 7 (6 num.) & UCI (removed unique model name and \\
& & & linear regression prediction of target) \\
Auto-Mpg & 398 & $7(4$ num.) & Torgo \\
Auto-Price & 159 & 15 (14 num.) & Torgo \\
Abalone & 4177 & 8 (7 num.) & UCI \\
Servo & 167 & 4 (0 num.) & UCI \\
Wisconsin & 198 & $34(33$ num.) & UCI (prognostic dataset) \\
Kin8NM & 8192 & 8 (8 num.) & Torgo \\
Puma8NH & 8192 & 8 (8 num.) & Torgo \\
Puma32H & 8192 & 32 (32 num.) & Torgo \\
Bank8FM & 8192 & 8 (8 num.) & Torgo \\
Bank32NH & 8192 & 32 (32 num.) & Torgo \\
\hline
\end{tabular}

Table 4: Accuracy for real world data sets.

\begin{tabular}{lrlrl}
\hline & \multicolumn{2}{c}{ MAUVE } & \multicolumn{2}{c}{ M5 } \\
Data set & RRMSE (StdDev) & Wins & RRMSE (StdDev) & Wins \\
\hline Housing & $47.98 \%(8.78)$ & 8 & $46.56 \%(3.73)$ & 12 \\
Machines & $\mathbf{3 2 . 0 6 \% ( 3 . 0 3 )}$ & 19 & $37.34 \%(4.85)$ & 1 \\
Auto-Mpg & $36.73 \%(0.87)$ & 8 & $36.91 \%(0.95)$ & 12 \\
Auto-Price & $56.08 \%(7.72)$ & 1 & $\mathbf{4 2 . 1 6 \%}(3.62)$ & 19 \\
Abalone & $68.17 \%(2.10)$ & 9 & $67.25 \%(0.51)$ & 11 \\
Servo & $40.14 \%(3.31)$ & 17 & $42.81 \%(3.03)$ & 3 \\
Wisconsin & $103.69 \%(4.47)$ & 12 & $104.42 \%(2.77)$ & 8 \\
Kin8NM & $\mathbf{6 0 . 0 2 \% ( 1 . 1 9 )}$ & 20 & $66.47 \%(0.66)$ & 0 \\
Puma8NH & $\mathbf{5 6 . 9 4 \% ( 0 . 0 9 )}$ & 20 & $57.88 \%(0.15)$ & 0 \\
Puma32H & $28.31 \%(0.21)$ & 11 & $28.28 \%(0.19)$ & 9 \\
Bank8FM & $\mathbf{2 0 . 2 1 \% ( 0 . 0 9 )}$ & 19 & $20.42 \%(0.08)$ & 1 \\
Bank32NH & $\mathbf{6 7 . 6 2 \% ( 0 . 2 6 )}$ & 19 & $69.34 \%(0.88)$ & 1 \\
\hline
\end{tabular}

Table 5: Size and CPU times for real world data sets.

\begin{tabular}{lrlrlrl}
\hline & \multicolumn{4}{c}{ Size } & \multicolumn{3}{c}{ CPU times } \\
\cline { 2 - 7 } Data set & Leaves & Pars & Leaves & Pars & Secs & Secs \\
\hline Housing & $\mathbf{6}$ & 46 & 13 & 52 & 0.93 & 1.14 \\
Machines & $\mathbf{2}$ & 38 & 3 & $\mathbf{1 5}$ & 0.46 & 0.46 \\
Auto-Mpg & 3 & 32 & $\mathbf{2}$ & $\mathbf{1 7}$ & 0.75 & 0.69 \\
Auto-Price & $\mathbf{1}$ & $\mathbf{1 1}$ & 10 & 29 & 0.30 & 0.35 \\
Abalone & 12 & 87 & 13 & 79 & 4.92 & 4.97 \\
Servo & 5 & 37 & 5 & $\mathbf{2 1}$ & 0.24 & 0.27 \\
Wisconsin & 3 & 29 & 4 & $\mathbf{1 4}$ & 0.49 & 0.47 \\
Kin8NM & $\mathbf{6 2}$ & 473 & 173 & 688 & 94.10 & 89.41 \\
Puma8NH & $\mathbf{1 7}$ & $\mathbf{7 4}$ & 46 & 153 & 93.58 & 104.45 \\
Puma32H & $\mathbf{2 6}$ & $\mathbf{2 3 8}$ & 169 & 618 & 141.68 & 132.79 \\
Bank8FM & 23 & 170 & 24 & 145 & 110.76 & 86.18 \\
Bank32NH & 3 & 56 & 3 & 54 & 116.16 & 150.19 \\
\hline
\end{tabular}


Table 6: Summary of the comparison between MAuve and M5' on real world datasets. One method is said to be winning in accuracy if its RRMSE is smaller in $90 \%$ of the cases. One method is said to be winning in size if it results in a tree with one third less leaves.

\begin{tabular}{clccc}
\hline & & \multicolumn{3}{c}{ Accuracy } \\
& & Mauve wins & Equality & M5' wins \\
\hline \multirow{N}{*}{$*$} & Mauve wins & 3 & 2 & 1 \\
& Equality & 2 & 3 & 0 \\
& M5' wins & 0 & 1 & 0 \\
\hline
\end{tabular}

We can conclude that, from the point of view of minimizing the number of subregions in the input space where the same linear relation holds, the use of a simple regression based heuristic is in many cases useful for real world data. As can be seen from the induction times in Table 5, this heuristic comes at no additional computational cost.

In the following sections we examine whether this conclusion still holds in a different setting, e.g. when the pruning parameter is tuned, or when the smoothing operator is used.

\subsubsection{Tuning the pruning factor}

As discussed in Sect. 4.2.4 the two error estimates to be compared in the pruning phase are multiplied with a compensation factor that takes into account the number of parameters. In the calculation of the compensation factor a multiplier is used that was called the pruning factor. This parameter controls the size of the tree. The previous experiments were performed using M5's default value of 2 for this parameter. Since in MAUve the leaves contain linear models that may include all predictor variables (while M5' only includes the variables occurring in the pruned subtree), another value might be more appropriate, which might influence our experimental results. Therefore, the experiments were rerun, both for M5' and for MAUVE, while estimating an optimal value for the pruning factor using crossvalidation. We proceeded as follows. For each of the training sets in the tenfold crossvalidation, we performed an inner threefold crossvalidation to estimate the optimal value for that training set. This optimal value was then used to test the model on the remaining test data. This procedure was again performed 20 times. The values for the pruning factor that were tested are 1 , $2,3,4$, and 5 .

Table 7 shows error estimates. Model complexity is shown in Table 8. The number of leaves (parameters) is now the mean value of the number of leaves (parameters) of the model for each fold in the 20 tenfold crossvalidations. The tables show that, for both methods, the RRMSE value is not that much affected, but its variance tends to decrease. The size of the trees and the number of parameters also tend to decrease.

From Tables 7, 8, and 9 we learn that the relative performance of both systems is not much affected by the changed experimental setting. The only 
Table 7: Accuracy for real world data sets with optimal pruning factor.

\begin{tabular}{lrlrl}
\hline & \multicolumn{1}{c}{ MAUVE } & M5' & \\
Data set & RRMSE (StdDev) & Wins & RRMSE (StdDev) & Wins \\
\hline Housing & $48.42 \%(3.98)$ & 6 & $46.38 \%(4.34)$ & 14 \\
Machines & $\mathbf{3 2 . 1 5 \%}(2.96)$ & 19 & $37.25 \%(4.83)$ & 1 \\
Auto-Mpg & $37.23 \%(0.84)$ & 5 & $36.63 \%(0.74)$ & 15 \\
Auto-Price & $53.79 \%(5.75)$ & 1 & $\mathbf{4 2 . 4 3 \%}(3.35)$ & 19 \\
Abalone & $67.32 \%(1.15)$ & 14 & $67.43 \%(0.38)$ & 6 \\
Servo & $39.92 \%(3.40)$ & 14 & $42.50 \%(2.54)$ & 6 \\
Wisconsin & $106.17 \%(5.02)$ & 6 & $101.80 \%(3.54)$ & 14 \\
Kin8NM & $\mathbf{6 0 . 1 6 \%}(0.70)$ & 20 & $66.35 \%(0.60)$ & 0 \\
Puma8NH & $\mathbf{5 6 . 8 9 \%}(0.07)$ & 20 & $57.52 \%(0.15)$ & 0 \\
Puma32H & $28.27 \%(0.18)$ & 0 & $\mathbf{2 7 . 4 6 \%}(0.13)$ & 20 \\
Bank8FM & $\mathbf{2 0 . 2 6 \%}(0.09)$ & 18 & $20.43 \%(0.08)$ & 2 \\
Bank32NH & $\mathbf{6 7 . 5 4 \% ( 0 . 1 7 )}$ & 19 & $68.02 \%(0.26)$ & 1 \\
\hline
\end{tabular}

Table 8: Model size for real world data sets with optimal pruning factor.

\begin{tabular}{lrlrl}
\hline & \multicolumn{2}{c}{ MAUVE } & \multicolumn{2}{c}{ M5 } \\
Data set & Leaves & Pars & Leaves & Pars \\
\hline Housing & $\mathbf{4 . 8 6}$ & 40.51 & 10.81 & 43.56 \\
Machines & $\mathbf{2 . 6 6}$ & 35.79 & 4.50 & $\mathbf{1 7 . 3 3}$ \\
Auto-Mpg & 2.56 & 26.05 & 2.38 & 18.30 \\
Auto-Price & $\mathbf{2 . 1 4}$ & 19.60 & 5.46 & 17.65 \\
Abalone & 8.43 & 57.92 & 6.19 & 39.15 \\
Servo & 4.03 & 28.47 & 4.28 & 19.59 \\
Wisconsin & $\mathbf{1 . 7 1}$ & 23.21 & 3.38 & $\mathbf{1 2 . 6 6}$ \\
Kin8NM & $\mathbf{5 4 . 0 9}$ & 384.80 & 103.54 & 442.22 \\
Puma8NH & $\mathbf{1 4 . 3 1}$ & $\mathbf{6 1 . 3 9}$ & 26.02 & 102.88 \\
Puma32H & $\mathbf{1 7 . 5 7}$ & $\mathbf{1 4 2 . 5 5}$ & 57.20 & 296.13 \\
Bank8FM & 23.58 & 173.26 & 27.19 & 157.13 \\
Bank32NH & 3.53 & 59.84 & 3.72 & 49.91 \\
\hline
\end{tabular}

changes concern the Puma32H dataset, for which M5' becomes superior in accuracy, and the Auto-Mpg data, for which the size of both models becomes equal.

\subsubsection{Using the smoothing operator}

A last experiment investigates the validity of our results in a setting where smoothing is used. At the same time, we examine whether smoothing improves the predictive performance of MAUve, as it does for standard M5' [17]. The pruning factor was reset to its default value in this experiment. Since smoothing is a post-processing procedure it has no effect on the size of the tree. Therefore, we only report predictive performances (Table 10). For both systems, we see high improvements in RRMSE, up to an improvement of $7.11 \%$ for the Auto-Price dataset for MAUvE.

Concerning the relative performance of both systems, we see no important changes in the summary table (Table 11). The only difference w.r.t. not using the smoothing operator again concerns the Puma32H dataset, for which M5' wins 
Table 9: Summary of the comparison between MAuve and M5' on real world datasets with optimal pruning factor. One method is said to be winning in accuracy if its RRMSE is smaller in $90 \%$ of the cases. One method is said to be winning in size if it results in a tree with one third less leaves.

\begin{tabular}{clccc}
\hline & & \multicolumn{3}{c}{ Accuracy } \\
& & Mauve wins & Equality & M5' wins \\
\hline \multirow{N}{*}{} & Mauve wins & 3 & 2 & 2 \\
$\tilde{\omega}^{2}$ & Equality & 2 & 3 & 0 \\
& M5' wins & 0 & 0 & 0 \\
\hline
\end{tabular}

Table 10: Accuracy for real world data sets when smoothing is used.

\begin{tabular}{lrlrl}
\hline & \multicolumn{1}{c}{ MAUVE } & M5' & \\
Data set & RRMSE (StdDev) & Wins & RRMSE (StdDev) & Wins \\
\hline Housing & $43.55 \%(6.72)$ & 10 & $44.13 \%(2.66)$ & 10 \\
Machines & $\mathbf{3 0 . 8 6 \%}(3.02)$ & 19 & $33.74 \%(4.05)$ & 1 \\
Auto-Mpg & $36.08 \%(0.63)$ & 10 & $36.25 \%(0.51)$ & 10 \\
Auto-Price & $48.97 \%(3.70)$ & 1 & $\mathbf{4 1 . 0 7 \%}(2.16)$ & 19 \\
Abalone & $66.44 \%(0.62)$ & 14 & $66.74 \%(0.41)$ & 6 \\
Servo & $39.37 \%(2.47)$ & 11 & $40.30 \%(1.83)$ & 9 \\
Wisconsin & $100.91 \%(3.22)$ & 4 & $97.86 \%(1.57)$ & 16 \\
Kin8NM & $\mathbf{5 8 . 8 7 \%}(0.84)$ & 20 & $63.68 \%(0.49)$ & 0 \\
Puma8NH & $\mathbf{5 6 . 8 1 \% ( 0 . 0 6 )}$ & 20 & $57.26 \%(0.11)$ & 0 \\
Puma32H & $28.17 \%(0.17)$ & 0 & $\mathbf{2 7 . 0 7 \%}(0.09)$ & 20 \\
Bank8FM & $\mathbf{2 0 . 1 0 \% ( 0 . 0 8 )}$ & 18 & $20.25 \%(0.05)$ & 2 \\
Bank32NH & $\mathbf{6 7 . 5 2 \% ( 0 . 1 9 )}$ & 20 & $68.33 \%(0.33)$ & 0 \\
\hline
\end{tabular}

in accuracy.

\subsection{An extended comparison}

In this last experiments section, we want to investigate where MAUVE is positioned with respect to other model tree builders. For each of the four options discussed in section 3 we choose a representative system:

1. M5'

2. MAUVE

3. Treed Regression [1]

4. RETIS [8]

In order to be able to compare the results (including induction times) in an objective manner, we also implemented these last two systems in Weka. In fact, we used MAUVE's implementation and only adapted its QUALITY function (the QUALITY functions of TREED REGRESSION and RETIS are shown in Figures 6 and 5, respectively). Therefore, a difference between TREED REGRESSION and our implementation is that in the latter, multiple linear models 
Table 11: Summary of the comparison between MAUvE and M5' on real world datasets, when the smoothing operator is used. One method is said to be winning in accuracy if its RRMSE is smaller in $90 \%$ of the cases. One method is said to be winning in size if it results in a tree with one third less leaves.

\begin{tabular}{clccc}
\hline & & \multicolumn{3}{c}{ Accuracy } \\
& & Mauve wins & Equality & M5' wins \\
\hline \multirow{N}{N}{} & Mauve wins & 3 & 1 & 2 \\
E & Equality & 2 & 3 & 0 \\
& M5' wins & 0 & 1 & 0 \\
\hline
\end{tabular}

are used in the leaves (while TREED REGRESSION uses simple linear models). This allows for a better comparison with the other methods. We denote our Weka-implementation of Treed Regression and Retis by TR' and Retis' respectively. As in MAUVE (and in M5') the calculations of the residual standard deviations are performed incrementally in TR', in order to evaluate the set of candidate splits in an efficient way. In the implementation of RETIS', we used the incremental formulae proposed by Torgo [16].

For completeness, we also included a standard regression tree inducer in our comparison. For this purpose we used M5' with the option to induce a normal regression tree instead of a model tree. This method is called REGTREE in the comparison. Remark that REGTREE is also an option 1 system, since it employs the same heuristic function as M5'.

For these experiments, both the pruning factor tuning and the smoothing operator were used. This gives us some kind of optimal experimental setup. The experiments were conducted on the same real world data sets of the previous section. In the tables we highlight the best results for each data set, without adding any statistical significance. Again, each experiment was performed 20 times and the results are averaged.

Table 12 shows the RRMSE for the different systems. We see that, except for the Wisconsin data set, REGTREE always performs worst. Hence, the benefit of using model trees instead of standard regression trees is clearly shown. For the model tree systems, however, differences are smaller and, although MAUVE and ReTis' have the most winners, no clear conclusions can be drawn.

Tables 13 and 14 show the average number of leaves and parameters, respectively. We see in Table 13 that REGTREE and M5' build overly large trees, as discussed in previous sections. The other systems are better matched. The differences in model size are reduced as we look at the number of parameters (Table 14). Note that for the standard regression trees, if the number of leaves is $n$, then the number of parameters is $2 n-1$.

If we compare Tables 13 and 14 to Table 8, we see large discrepancies in model size. The only difference in experimental setup is that smoothing is used in this last experiment. This suggests that the smoothing operator favours large trees. Remark that smoothing itself does not alter the tree size. When tuning the pruning factor, however, smoothed trees are compared in each fold of the 
Table 12: Accuracy for the different systems.

\begin{tabular}{|c|c|c|c|c|c|}
\hline & REGTREE & M5 & MAUVE & TR' & RETIS' \\
\hline Data set & RRMSE (StD) & RRMSE (StD) & RRMSE (StD) & RRMSE (StD) & RRMSE (StD) \\
\hline Housing & $52.22 \%(0.93)$ & $44.02 \%(2.80)$ & $44.15 \%(1.80)$ & $\mathbf{3 8 . 9 7 \%}(1.41)$ & $40.10 \%(1.95)$ \\
\hline Machines & $71.80 \%(1.55)$ & $33.65 \%(4.09)$ & $\mathbf{3 0 . 9 2 \%}(2.97)$ & $40.15 \%$ & $36.92 \%(4.73)$ \\
\hline Auto-Mpg & $46.63 \%(0.75)$ & $36.34 \%(0.51)$ & $35.99 \%(0.74)$ & $\mathbf{3 5 . 4 1 \%}(0.99)$ & $37.95 \%(1.73)$ \\
\hline Auto-Price & $56.90 \%(1.26)$ & $\mathbf{4 0 . 8 0 \%}(2.35)$ & $49.11 \%(2.77)$ & $46.84 \%(4.92)$ & $53.05 \%(8.71)$ \\
\hline Abalone & $69.77 \%(0.32)$ & $67.06 \%(0.50)$ & $\mathbf{6 6 . 3 6 \%}(0.57)$ & $67.04 \%(0.21)$ & $66.80 \%(0.62)$ \\
\hline Servo & $59.57 \%(1.18)$ & $39.93 \%(1.53)$ & $\mathbf{3 8 . 8 2 \%}(2.37)$ & $38.89 \%(2.43)$ & $39.27 \%(2.13)$ \\
\hline Wisconsin & $85.69 \%(1.57)$ & $\mathbf{8 3 . 4 2 \%}(2.05)$ & $89.05 \%(4.49)$ & $87.51 \%(4.36)$ & $90.29 \%(2.43)$ \\
\hline Kin8NM & $69.91 \%(0.31)$ & $62.92 \%(0.44)$ & $57.76 \%(0.57)$ & $53.47 \%(0.33)$ & $49.23 \%(0.55)$ \\
\hline Puma8NH & $58.63 \%(0.13)$ & $57.29 \%(0.11)$ & $\mathbf{5 6 . 8 2 \%}(0.06)$ & $56.88 \%(0.06)$ & $56.93 \%(0.07)$ \\
\hline Puma32H & $28.48 \%(0.10)$ & $27.09 \%(0.09)$ & $28.19 \%(0.16)$ & $24.88 \%(0.07)$ & $\mathbf{2 2 . 0 9 \%}(0.08)$ \\
\hline Bank8FM & $25.64 \%(0.09)$ & $20.15 \%(0.07)$ & $20.04 \%(0.07)$ & $20.62 \%(0.15)$ & $19.64 \%(0.06)$ \\
\hline Bank32NH & $75.10 \%(0.24)$ & $67.93 \%(0.17)$ & $67.49 \%(0.15)$ & $67.47 \%(0.16)$ & $\mathbf{6 7 . 4 1 \%}(0.29)$ \\
\hline
\end{tabular}

Table 13: Number of leaves for the different systems.

\begin{tabular}{rccccc}
\hline & $\begin{array}{c}\text { REGTREE } \\
\text { Leaves }\end{array}$ & $\begin{array}{c}\text { M5' } \\
\text { Leaves }\end{array}$ & $\begin{array}{c}\text { MAUVE } \\
\text { Leaves }\end{array}$ & $\begin{array}{c}\text { TR' } \\
\text { Leaves }\end{array}$ & $\begin{array}{c}\text { RETIS' } \\
\text { Leaves }\end{array}$ \\
\hline Housing & 36.29 & 18.77 & 7.39 & 10.44 & $\mathbf{6 . 1 8}$ \\
Machines & 15.08 & 5.02 & $\mathbf{2 . 6 6}$ & 2.85 & 3.49 \\
Auto-Mpg & 26.74 & 3.32 & 4.15 & 6.17 & $\mathbf{3 . 3 1}$ \\
Auto-Price & 10.59 & 7.50 & $\mathbf{2 . 3 4}$ & 2.95 & 2.99 \\
Abalone & 105.87 & 20.48 & 12.84 & $\mathbf{5 . 2 9}$ & 5.65 \\
Servo & 10.34 & 4.66 & 4.39 & $\mathbf{4 . 1 5}$ & 4.52 \\
Wisconsin & 16.33 & 11.06 & 2.88 & 2.60 & $\mathbf{1 . 4 8}$ \\
Kin8NM & 576.03 & 400.80 & $\mathbf{1 3 5 . 6 0}$ & 272.49 & 201.59 \\
Puma8NH & 205.95 & 38.83 & 16.12 & 19.09 & $\mathbf{1 4 . 1 6}$ \\
Puma32H & 759.25 & 147.83 & $\mathbf{1 8 . 8 1}$ & 38.83 & 36.67 \\
Bank8FM & 451.78 & 64.71 & $\mathbf{3 1 . 7 1}$ & 64.16 & 33.38 \\
Bank32NH & 267.33 & 3.82 & $\mathbf{3 . 6 6}$ & 3.69 & 4.21 \\
\hline
\end{tabular}

tenfold crossvalidation. For each fold, the smoothed tree with the lowest error (determined by an inner threefold crossvalidation) is chosen. Since smoothing can be seen as somewhat reducing the effect of a split, larger trees may be chosen.

Induction times for building a single tree are shown in Table 15. A first observation is that the induction times of RETIS' may be too high for many practical applications. For instance, for the Puma32H dataset, building one tree took us over six hours (on an Intel Pentium4 system with a $2.0 \mathrm{GHz} \mathrm{CPU}$ ). If one wants to tune the pruning factor using our method, in total 160 trees are built (although most of them on smaller parts of the data). A second observation is that, although we would expect REGTREE to be the fastest (since it does not build regression models), in three cases MAuve is faster. This fact, together with the sizes of the pruned trees in Table 13 suggests that MaUvE's tree building procedure in general stops earlier. A last observation concerns the Bank8FM dataset, where TR' has a shorter induction time than MAuve, 
Table 14: Number of parameters for the different systems.

\begin{tabular}{rccccc}
\hline & $\begin{array}{c}\text { REGTREE } \\
\text { Pars }\end{array}$ & $\begin{array}{c}\text { M5' } \\
\text { Pars }\end{array}$ & $\begin{array}{c}\text { Mauve } \\
\text { Pars }\end{array}$ & $\begin{array}{c}\text { TR' } \\
\text { Pars }\end{array}$ & $\begin{array}{c}\text { RETIS' } \\
\text { Pars }\end{array}$ \\
\hline Housing & 71.58 & 63.70 & $\mathbf{5 6 . 4 8}$ & 73.70 & 56.49 \\
Machines & 29.17 & $\mathbf{1 8 . 7 4}$ & 35.83 & 33.34 & 37.80 \\
Auto-Mpg & 52.49 & $\mathbf{2 0 . 8 7}$ & 36.95 & 46.94 & 36.13 \\
Auto-Price & $\mathbf{2 0 . 1 8}$ & 22.18 & 21.52 & 28.73 & 34.30 \\
Abalone & 210.74 & 83.38 & 80.08 & $\mathbf{3 4 . 7 2}$ & 41.24 \\
Servo & $\mathbf{1 9 . 6 8}$ & 20.53 & 30.85 & 28.40 & 29.90 \\
Wisconsin & 31.67 & 30.31 & 38.58 & 34.83 & $\mathbf{2 7 . 0 7}$ \\
Kin8NM & 1151.07 & 1184.45 & $\mathbf{8 4 3 . 6 8}$ & 1464.77 & 1523.98 \\
Puma8NH & 410.91 & 135.31 & 68.56 & 77.51 & $\mathbf{6 3 . 6 3}$ \\
Puma32H & 1517.51 & 531.18 & $\mathbf{1 5 3 . 3 1}$ & 313.21 & 380.91 \\
Bank8FM & 902.56 & 284.49 & $\mathbf{2 2 5 . 1 6}$ & 375.70 & 250.79 \\
Bank32NH & 533.67 & $\mathbf{5 0 . 5 4}$ & 61.80 & 60.09 & 73.63 \\
\hline
\end{tabular}

Table 15: Total CPU time used for the different systems.

\begin{tabular}{rccccc}
\hline & $\begin{array}{c}\text { REGTREE } \\
\text { Secs }\end{array}$ & $\begin{array}{c}\text { M5' } \\
\text { Secs }\end{array}$ & $\begin{array}{c}\text { MAUVE } \\
\text { Secs }\end{array}$ & $\begin{array}{c}\text { TR' } \\
\text { Secs }\end{array}$ & $\begin{array}{c}\text { RETIS' } \\
\text { Secs }\end{array}$ \\
\hline Housing & 0.91 & 1.05 & $\mathbf{0 . 8 6}$ & 1.39 & 19.47 \\
Machines & $\mathbf{0 . 3 5}$ & 0.37 & 0.38 & 0.92 & 36.40 \\
Auto-Mpg & $\mathbf{0 . 5 7}$ & 0.61 & 0.67 & 1.12 & 39.59 \\
Auto-Price & $\mathbf{0 . 2 5}$ & 0.31 & 0.29 & 0.35 & 4.87 \\
Abalone & $\mathbf{3 . 8 4}$ & 4.88 & 4.78 & 7.35 & 605.04 \\
Servo & 0.20 & 0.23 & $\mathbf{0 . 1 6}$ & 0.27 & 3.23 \\
Wisconsin & $\mathbf{0 . 3 5}$ & 0.40 & 0.42 & 0.70 & 34.53 \\
Kin8NM & $\mathbf{8 4 . 3 8}$ & 86.90 & 89.13 & 91.51 & 1976.87 \\
Puma8NH & 95.75 & 101.14 & $\mathbf{9 1 . 5 7}$ & 102.06 & 2641.36 \\
Puma32H & $\mathbf{8 8 . 6 3}$ & 130.16 & 139.43 & 224.21 & 24175.54 \\
Bank8FM & 83.61 & 84.34 & 107.62 & $\mathbf{8 1 . 3 4}$ & 2835.64 \\
Bank32NH & $\mathbf{8 9 . 5 2}$ & 147.55 & 114.69 & 177.73 & 23561.74 \\
\hline
\end{tabular}

although its pruned tree is twice as large. Given the fact that TR's tree building procedure is more complex, we conclude that MAUVE's pruning procedure for this dataset takes much more time. Because of these two last observations we also measured the CPU time needed for the GROW_TREE function (see Fig. 1) for each system, i.e. the time needed to grow the initial tree, before pruning takes place and before any regression model is built in the leaves. Table 16 shows the results. There we see that MAUvE always has the shortest CPU time (except for one case). Since the time needed for pruning is relatively stable compared to the time needed for growing the tree, it follows that the relative time spent in the pruning phase is much smaller for RETIS' (3.4\% on average) than for, e.g., MAUve (92.4\% on average).

This extensive comparison of different model tree systems is summarized in Fig. 10. We left out the results for REgTree and take M5' as a base reference. The results of the other model tree inducers are plotted against this base reference. We conclude that going from option 1 (represented by M5') 
Table 16: CPU time used for growing the tree for the different systems.

\begin{tabular}{rccccc}
\hline & $\begin{array}{c}\text { RegTrEe } \\
\text { Secs }\end{array}$ & $\begin{array}{c}\text { M5' } \\
\text { Secs }\end{array}$ & $\begin{array}{c}\text { MaUve } \\
\text { Secs }\end{array}$ & $\begin{array}{c}\text { TR' } \\
\text { Secs }\end{array}$ & $\begin{array}{c}\text { ReTIS' } \\
\text { Secs }\end{array}$ \\
\hline Housing & 0.10 & 0.10 & $\mathbf{0 . 0 6}$ & 0.53 & 18.56 \\
Machines & 0.09 & 0.09 & $\mathbf{0 . 0 5}$ & 0.53 & 36.01 \\
Auto-Mpg & 0.10 & 0.10 & $\mathbf{0 . 0 7}$ & 0.66 & 39.03 \\
Auto-Price & 0.05 & 0.05 & $\mathbf{0 . 0 3}$ & 0.15 & 4.67 \\
Abalone & 0.70 & 0.72 & $\mathbf{0 . 6 9}$ & 3.75 & 600.42 \\
Servo & 0.03 & 0.03 & $\mathbf{0 . 0 2}$ & 0.12 & 3.05 \\
Wisconsin & 0.08 & 0.08 & $\mathbf{0 . 0 4}$ & 0.36 & 32.07 \\
Kin8NM & 1.53 & 1.54 & $\mathbf{1 . 4 7}$ & 7.08 & 1866.99 \\
Puma8NH & 1.69 & 1.69 & $\mathbf{1 . 4 9}$ & 7.78 & 2486.98 \\
Puma32H & 6.61 & 6.63 & $\mathbf{6 . 0 0}$ & 80.09 & 24021.03 \\
Bank8FM & $\mathbf{1 . 5 1}$ & 1.53 & 1.83 & 6.80 & 2742.07 \\
Bank32NH & 8.24 & 8.26 & $\mathbf{5 . 5 0}$ & 71.60 & 23418.22 \\
\hline
\end{tabular}

to option 2 (represented by MAUve) leads to a large drop in the number of leaves while costing nothing w.r.t. accuracy or induction time. Going to more sophisticated systems in general does not add any noticeable improvements in accuracy or model size, while giving up on efficiency.

\section{Conclusions}

The contributions of this paper can be summarized as follows. First, we have studied the behaviour of variance as a heuristic for building model trees. While the inappropriateness of variance for this task, in itself, was pointed out in earlier work $[8,10]$, no investigation was performed on exactly how this influences the quality of the induced trees. Our results show that it mainly influences the explanatory power of the tree, rather than its predictive power.

Since explanatory power is often mentioned as an important advantage of trees over black-box models, it is worthwhile to try to improve it. Therefore, as a second contribution, we have presented an approach that does indeed induce model trees with better explanatory power, in the sense that shorter trees are built. This approach has a complexity that is linear in the number of attributes, and as such differs from the complexity of the most efficient approaches only with a constant factor. Currently existing alternative approaches towards building better trees (such as Retis or SMOTI) have a complexity that is at least quadratic.

Our experimental validation confirms that the heuristic we propose yields simpler trees with equal predictive accuracy in cases where there is a piecewise linear relation between the target and the input variables. Moreover, the results suggest that this kind of behaviour often occurs in real world data. Finally, our analysis shows that using more sophisticated approaches on average does not yield improvements in accuracy or model size. 

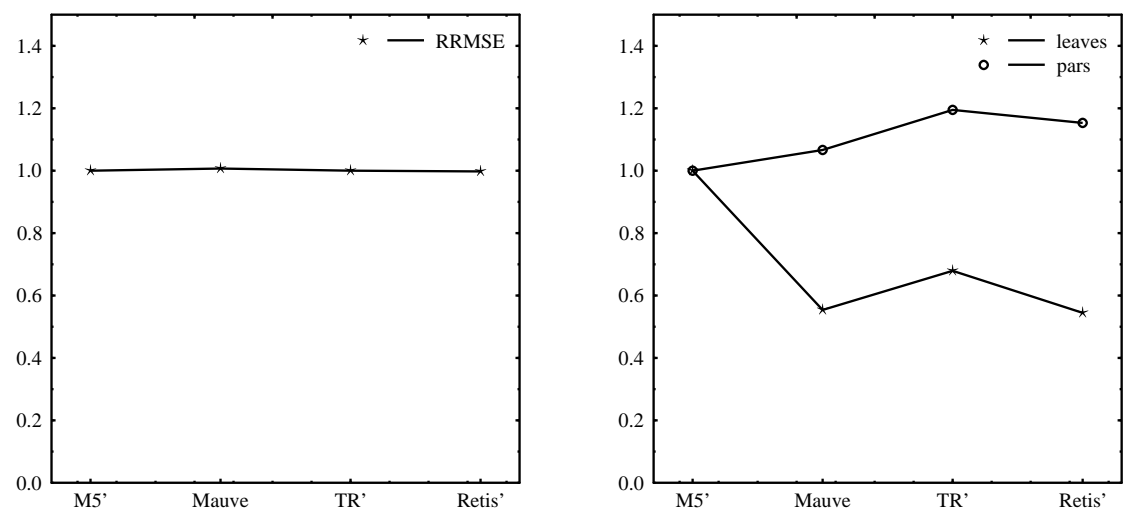

(a)

(b)

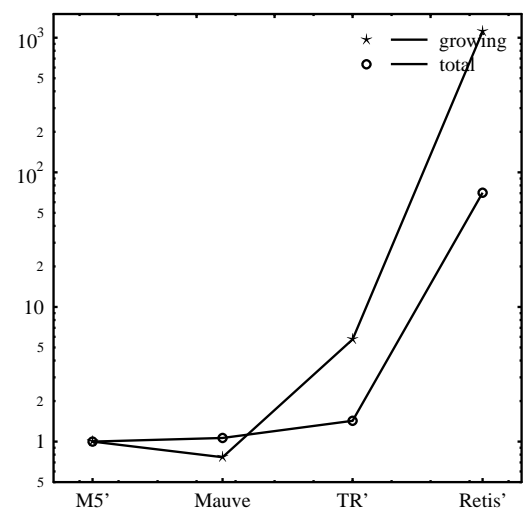

(c)

Figure 10: Summary of the comparison of different model tree inducers. (a) RRMSE (b) Model Size (c) Induction times (logarithmic scale). 


\section{Acknowledgements}

Celine Vens is supported by the F.W.O. project on Probabilistic-Logical Learning. Hendrik Blockeel is Postdoctoral Fellow of the Fund for Scientific Research - Flanders (Belgium) (F.W.O.-Vlaanderen). The authors also want to thank the reviewers for their valuable comments and suggestions.

\section{References}

[1] W.P. Alexander and S.D. Grimshaw. Treed regression. Journal of Computational and Graphical Statistics, 5:156-175, 1996.

[2] L. Breiman, J.H. Friedman, R.A. Olshen, and C.J. Stone. Classification and Regression Trees. Wadsworth, Belmont, 1984.

[3] C.E. Brodley and P.E. Utgoff. Multivariate decision trees. Machine Learning, 19:45-77, 1995.

[4] P. Chaudhuri, M Huang, W. Loh, and R. Yao. Piecewise-polynomial regression trees. Statistica Sinica, 4:143-167, 1994.

[5] T. G. Dietterich. Approximate statistical tests for comparing supervised classification learning algorithms. Neural Computation, 10(7):1895-1924, 1998.

[6] A. Dobra and J. Gehrke. Secret: A scalable linear regression tree algorithm. In Proceedings of the 8th ACM SIGKDD International Conference on Knowledge Discovery and Data Mining, 2002.

[7] J. Friedman. Multivariate adaptive regression splines (with discussion). Annals of Statistics, 19:1-142, 1991.

[8] A. Karalic. Employing linear regression in regression tree leaves. In European Conference on Artificial Intelligence, pages 440-441, 1992.

[9] W.-Y. Loh. Regression trees with unbiased variable selection and interaction detection. Statistica Sinica, 12:361-386, 2002.

[10] D. Malerba, F. Esposito, M. Ceci, and A. Appice. Top-down induction of model trees with regression and splitting nodes. IEEE Transactions on Pattern Analysis and Machine Intelligence, 26(5):612-625, 2004.

[11] C.J. Merz and P.M. Murphy. UCI repository of machine learning databases

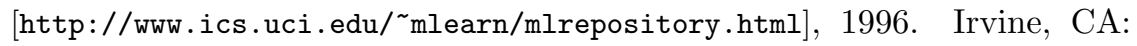
University of California, Department of Information and Computer Science.

[12] S.K. Murthy and S. Salzberg. Lookahead and pathology in decision tree induction. In Proceedings of the 14th International Joint Conference on Artificial Intelligence, pages 1025-1031. Morgan Kaufmann, 1995. 
[13] J. R. Quinlan. C4.5: Programs for Machine Learning. Morgan Kaufmann series in Machine Learning. Morgan Kaufmann, 1993.

[14] J.R. Quinlan. Learning with continuous classes. In Proceedings of the 5th Australian Joint Conference on Artificial Intelligence, pages 343-348. World Scientific, Singapore, 1992.

[15] Luís Torgo. Functional models for regression tree leaves. In Proceedings of the 14th International Conference on Machine Learning, pages 385-393. Morgan Kaufmann, 1997.

[16] Luís Torgo. Computationally efficient linear regression trees. In Proceedings of the 8th Conference of the International Federation of Classification Societies. Springer, 2002.

[17] Y. Wang and I.H. Witten. Inducing model trees for continuous classes. In Proc. of the 9th European Conf. on Machine Learning Poster Papers, pages 128-137, 1997.

[18] S. Weisberg. Applied linear regression. John Wiley \& Sons, 1980.

[19] I. Witten and E. Frank. Data Mining: Practical Machine Learning Tools and Techniques with Java Implementations. Morgan Kaufmann, 1999. 